\title{
La psychiatrie de liaison
}

\author{
Blanche MASSARI*
}

* Praticien

Hospitalier,

Service

de pédopsychatrie,

Centre Hospitalier

Intercommuna

40 , avenue

de Verdun, 94010

Créteil, france.

massariblanche@

yahoo.tr

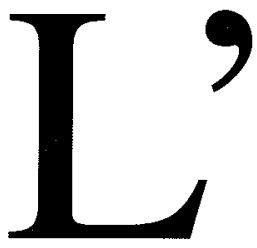

écrit se fonde sur le terrain, sinon il manque de substance. Depuis presque quinze ans, j'ai travaillé en tant que pédopsychiatre en hôpital général, que ce soit à Saint-Nazaire, à Melun et désormais au Centre Hospitalier Intercommunal de Créteil. Je me rends compte qu'il s'agit-là pour moi d'un choix délibéré. Pourtant, auparavant lors de mon internat et clinicat, $\mathrm{j}$ ' avais, dans nos hôpitaux universitaires (à Hérold dans le service du Pr Dugas, et à la Salpêtrière dans le service du Pr Duché), eu un avant-goût amer des possibles liens avec les autres disciplines, en particulier bien sûr la pédiatrie. À Hérold, aucun lien n'existait, chaque unité fonctionnait de façon autarcique, voire méfiante, ce qui était fort étonnant, les psychiatres du service ayant souvent une solide connaissance en pédiatrie, certains étaient même d'anciens pédiatres. Peut-être qu'alors, dans la décennie 1970 , on tournait sa veste sans regarder en arrière, on ne pouvait garder les liens dans une nouvelle identité. A la Salpêtrière, c'était le même clivage complet. Pourtant, j'avais assuré les urgences pour les adolescents et travaillais avec Daniel Sibertin-Blanc, à l'unité d'hospitalisation de « court séjour » justement après un accueil en urgence. J'étais aussi chargée de liaison au sens large (y compris d'ailleurs dans les services adultes): en un an, je ne fus appelée qu'une fois en pédiatrie... l'enfant n'était pas là mais «parti à la radio », ses parents non prévenus. Après trois essais, et sans pouvoir rencontrer le pédiatre demandeur, j'ai émis l'hypothèse que l'enfant, dont on craignait un retrait autistique, pouvait souffrir d'être séparé de sa mère et qu'il fallait vérifier son audition. Malgré mon désir, je n'ai jamais pu rencontrer le pédiatre, l'enfant est allé mieux au retour de sa mère retenue par son clan de gitans, une surdité importante fut diagnostiquée.

À l'époque, dans ma fougue juvénile, je tempêtais contre le manque d'intuition et de bon sens, le manque de respect pour mon travail. Maintenant, avec d'autres expériences fructueuses, je sais bien que, tout simplement, quand il n'y a pas un fort désir partagé de travail en commun, aucune collaboration ne peut exister. Je sais aussi qu'un travail clinique sans lien et sans dialogue, sans histoire commune, est vide voire nuisible.

Mes colères, pour justifiées qu'elles fussent, tombèrent dans le vide. On ne peut bâtir sur si peu, mais pourtant une situation clinique partagée peut être initiatrice de part et d'autre. À Montesson, qui entre, 1980 et 1990, n'est pas un hôpital général mais encore hôpital psychiatrique pour enfants, quelques liens furent tissés avec les pédiatres de SaintGermain-en-Laye ; mais chacun restait alors à distance. Quant au pédiatre de l'institution, il n'intervenait que pour les fièvres, bobos et vaccinations. Le travail avec l'hôpital de Saint-Germain s'est beaucoup intensifié depuis la création, il y a une quinzaine d'années, d'une unité d'hospitalisation mèrebébé

J'ai passé la décennie suivante à SaintNazaire. Le secteur de pédopsychiatrie y était déjà bien équipé : deux CMP aux extrémités (à Guerande et à Pornic), un AFT, deux hôpitaux de jour de 15 places.

Le paradoxe était qu'il n'y avait pas, à SaintNazaire, de consultation dépendante du secteur. Mais deux autres lieux très actifs existaient : un CMPP et un CAMSP d'une part, et l'OCHS, d'autre part (Office central d'hygiène sociale, devenu une consultation de pédopsychiatrie généraliste). 
Nos prédécesseurs avaient déjà largement ouvert les liens avec le service de pédiatrie. Les pédiatres de leur côté avaient aussi avancé leurs pions. Le Dr Menu avait demandé fort longtemps auparavant la création dans son service de pédiatrie d'un poste de psychologue dont il était persuadé de l'intérêt, et de la nécessité. Ce poste a été très heureusement maintenu jusqu'à présent.

L'occasion de création de nouveaux projets est à saisir lorsqu'un nouveau chef de service est nommé. Le Dr Gloanec avait créé un nouvel hôpital de jour et l'AFT, élargissant les premières structures du secteur ouvert par G. Bourcier à la fin des années 1970.

La mission pour les praticiens en place et les nouveaux arrivants fut de renforcer les structures existantes et d'en créer de nouvelles (avec le nouveau chef de secteur: B. de La Gorce). Le choix fut d'axer l'effort sur les âges extrêmes en pédopsychiatrie, c'est-à-dire créer dans la ville une autre consultation d'une part pour les adolescents, d'autre part pour les tout petits qui, avant 18 mois, étaient peu reçus, même au CAMS.

Ce projet fut soutenu par l'hôpital et les tutelles et ces deux consultations, officiellement ouvertes en 1994, eurent leur histoire liée dès leur création avec le travail en pédiatrie.

Déjà, un peu plus tôt, les liens s'étaient renforcés, par la volonté commune des deux services, au moins au début autour des «grands 》 enfants, puis bientôt des petits et de la néonatologie. Il s'agit là d'un modèle de collaboration mixte où les deux services ont leur autonomie, avec chacune leur personnel (qui pour la pédopsychiatrie s'est étoffé pour des consultations spécifiques mais reste insuffisant).

L'équipe de pédopsychiatrie, qui est la même que celle des consultations pour adolescents et pour petits, intervient en pédiatrie.

Le psychiatre n'intervient pas seulement à la demande. Un temps-plein est nécessaire (pour deux tiers pour les adolescents, pour un tiers pour les petits, avec deux PH différents). Le psychiatre n'intervient pas seul mais en équipe avec le soutien du chef de secteur (voir les articles de C. Haulle et de B. De La Gorce).

Des synthèses communes sont par ailleurs organisées.

Depuis 1998, une unité d'hospitalisation pédopsychiatrique a été officiellement créée en pédiatrie avec des personnels des deux services (ce qui se faisait auparavant de façon tacite mais l'aval du SROS renforce le projet).

Le Dr Picherot, chef de service de pédiatrie fut pour beaucoup dans la mise en place de ce travail en commun.
Le cadre institutionnel fut bien posé puisque, malgré son départ et celui de certains praticiens de notre service, le travail se poursuit Deux équipes se retrouvent ensemble, partagent les urgences et la réflexion. Il n'y a plus de face à face singulier ou au coup par coup même si des « urgences » existent, liées tant à la pathologie des enfants qu'à des comportements pas forcement de notre ressort mais impossibles à supporter dans ce cadre.

L'aval comme l'amont de ces hospitalisations étaient assurés par les CMP.

En « petite pédiatrie » et en néonatologie, d'autres expériences avaient eu lieu tant avec la psychologue du service, que de ses stagiaires.

Le pédiatre était ouvert, clair sur son rôle, ne mélangeant pas les espaces de chacun. Outre un partage direct régulier avec l'équipe pédiatrique, les indications plus ciblées du médecin étaient claires, observées avec acuité.

Un autre jeune collègue lui succéda. Il fallut recommencer les approches. Car dès que cette jeune pédiatre, par ailleurs rigoureuse et compétente, $m$ 'apercevait, elle partait en courant en commentant sa fuite. L'effet ludique suscité permit de nous apprivoiser l'une l'autre: la demande vint plus facile en colloque singulier qu'avec le reste de l'équipe soignante. Le chaleureux environnement de la néonatologie y était pour beaucoup, avec toujours en arrièreplan la présence d'un chef de service engagé. L'aventure nazairienne se poursuit toujours. À Melun, il me fallut ensuite retrouver un travail de psychiatrie de liaison moins institutionnalisé. Nous étions trois à intervenir, au départ «à la demande ». Cette demande s'est ensuite affinée ; notre venue hebdomadaire au staff de pédiatrie est devenue régulière, au point que notre rare absence y était remarquée. Là aussi, la continuité, l'essai de rendre le cadre plus rigoureux à pu permettre un réel travail en commun, même s'il n'existait pas une unité officielle d'hospitalisation.

Au CHIC, le travail en pédiatrie fut soutenu, fécond, prolongé. La difficulté vint du fait qu'il n'y avait pas, au sein du service de pédopsychiatrie, d'équipe spécifique d'intervenants; le travail de liaison reposait sur un praticien isolé. Les prises en charge en aval étaient périlleuses, les consultations encombrées et, de toute façon, non articulées avec un réel projet de liaison.

Lors de discussions avec V. Granboulan et J. Sarfaty, devenu depuis chef de service à la suite de B. Durand, le problème central repéré fut l'isolement de cette intervention.

Lorsque V. Granboulan cessa son activité en pédiatrie, elle réfléchit sur les autres expériences de liaison, qu'elle résuma en une note 
brève décrivant les différentes modalités de collaboration.

Des solutions très diverses en effet ont vu le jour :

- Des unités qualifiées de «mixtes », comme le service de Cholet où une équipe liée au secteur psychiatrique intervient en pédiatrie. C'est aussi le modèle nazairien ainsi que, à ses prémices, celui de Melun, parmi nos expériences personnelles.

Ce modèle a parfois suscité des échecs liés aux pathologies parfois trop lourdes des patients, ou au fait que l'hospitalisation en pédiatrie augmente le déni de tout processus psychique des enfants et leur famille.

Mais ce déni ne s'exerce-t-il pas aussi en psychiatrie ? N'est-ce pas justement tout le défi de notre travail, que de permettre de ranimer un système de représentation, de mise en pensée, en restant prudent car, parfois, le gel des affects peut se révéler un temps un bon moyen de défense voire de survie.

La deuxième difficulté est due à la confrontation de deux mondes différents: pédiatriepsychiatrie. La situation de patients psychiatriques hospitalisés en pédiatrie réalise souvent un équilibre précaire: nous sommes là sur un fil, mais un bon balancier peut parfois permettre de s'y retrouver (voir l'article de C. Haulle).

Certaines collaborations ont donc été des échecs mais nos exemples, en particulier celui de Saint-Nazaire, se sont montrés fructueux, vivants bien que difficiles. À Saint-Nazaire, les équipes, différentes dans leur histoire et dans leur culture, avaient officiellement leur place, et tout un code sur le rôle de chacun et les décisions était inscrit dans les deux services fédérés.

- Le modèle de deux institutions séparées, mais bien équipées. L'évaluation se fait aux urgences par un psychiatre, qui a le choix de la structure qui lui paraitra la mieux adaptée (hospitalisation en pédiatrie ou en psychiatrie).

- Le modèle des lits portes psychiatriques existe en psychiatrie adulte mais pas pour les enfants. Ce pourrait cependant être un modèle intéressant, en particulier pour les tentatives de suicide.

- Le modèle ambulatoire canadien où les psychiatres interviennent dès l'urgence, avec une prise en charge disponible en externe évitant souvent l'hospitalisation (qui reste cependant une solution possible si nécessaire).

- Le modèle pédiatrique pur où l'équipe est formée depuis longtemps à l'écoute, mais sans l'intervention d'un psychiatre (ni psychiatre rattaché au service, ni psychiatre extérieur). L'aspect psychiatrique est pris en charge par les psychologues du service. Les pédiatres restent alors seuls à poser les indications, la prise en charge, la sortie (modèle du service du Pr Alvin au CHU du Kremlin-Bicêtre).

Notons que, dans l'exemple des modèles mixtes, existaient toujours aussi des psychologues rattachés à la pédiatrie, avec alors toute l'ambiguité des compétences et des territoires : quand fait-on appel au psychiatre extérieur? C'est parfois une affaire d'indication, quand un suivi est pressenti. Mais c'est hélas plus souvent une histoire de jours de présence ou de pathologies plus explosives, plus dangereuses (plus «psychiatriques »?), en particulier quand se pose l'indication d'un traitement médicamenteux. Cette dernière situation soulève la question du rôle des psychologues de l'équipe psychiatrique par rapport à celui du psychologue de pédiatrie.

Réalisons là que rien n'est jamais acquis, l'avancée ne peut se faire qu'avec ouverture, disponibilité mais sans complaisance de part et d'autre. Heureusement, les ravages du psychiatre silencieux (qui sait ou est censé savoir) ont presque disparu mais pas forcément toujours sa représentation par certains «somaticiens », que ce soit peur, évitement, voire idéalisation tout aussi destructrice. Il faut partager, filtrer les informations en colloque singulier, en groupe : le minimum étant au moins de respecter le patient et son intimité, et les professionnels qui ont avec nous la responsabilité des soins.

Ces diverses expériences m'ont conduite à l'idée de coordonner ce dossier. Après l'exposé des différents modèles de collaboration et de notre histoire, plusieurs collègues ont accepté de participer à ce projet : faire le point sur les liens entre pédopsychiatre et pédiatre de façon non exhaustive.

Nous n'aborderons pas dans ce dossier les aspects singuliers des problèmes psychologiques posés pour telle maladie organique spécifique, tant d'ailleurs pour l'enfant et sa famille, mais nous décrirons les dispositifs de travail en commun, ainsi que les principes de la psychiatrie de liaison et son éthique.

Daniel Sibertin-Blanc, avec qui autrefois j'avais pu découvrir la pédopsychiatrie à l'hôpital général, me proposa deux écrits :

- un article de réflexion décalée sur le principe même du travail partagé et complémentaire où chacun garde sa spécificité : «La psychiatrie de liaison: une pratique de la transdisciplinarité, une éthique de la rencontre »; - un autre, à propos d'une expérience en maternité : «L'urgence : une dynamique dans 
la pratique de psychiatrie de liaison. Expérience en maternité ».

L'équipe nazairienne avec qui j'ai travaillé pendant presque une dizaine d'années a répondu à ma sollicitation avec enthousiasme.

C. Haulle, actuellement PH à Saint-Nazaire, et qui assure la liaison en pédiatrie, nous fait part dans son article, «La Blouse » (réflexions sur l'identité du psychiatre de liaison en pédiatrie), de la singularité des rôles. Sa conclusion m'a d'autant plus amusée que mes blouses blanches ont subi le même sort que les siennes !

G. Picherot, pédiatre à Saint-Nazaire et actuellement chef de service à Nantes, répondit avec élan à ma demande. Il souhaitait depuis longtemps mettre en forme, avec un peu de recul, son expérience en LoireAtlantique. Outre son rôle de chef de service à Saint-Nazaire, où il était responsable de l'unité des grands, il soutint fortement la collaboration avec le chef de secteur de pédopsychiatrie B. de La Gorce. Saint-Nazaire fut par ailleurs, grâce à son initiative, un site pilote pour l'accueil des enfants maltraités.

Son travail se poursuit au CHU de Nantes où l'équipe de psychiatres est uniquement destinée à la liaison.

B. de La Gorce, chef du secteur de pédopsychiatrie de Saint-Nazaire, évoquera la prise en charge de patientes anorexiques hospitalisées en pédiatrie en comparant les orientations cliniques et théoriques des hospitalisations en service spécialisé.

Charlotte Perdigon, psychiatre assistante, voulut bien jouer, avec prudence et élan mêlés, le rôle du candide : la jeune assistante, nouvellement promue psychiatre de liaison au CHIC dans l'unité d'adolescents du service de pédiatrie dont le Dr S. Lemerle est le pédiatre responsable, nous entraîne dans les émois et perplexité de son débarquement imprévu en pédiatrie. Ce regard est perspicace, plein d'intuition et d'humour. Nous aurons de la relève !

\title{
Communiqué
}

\section{L'attachement}

Applications thérapeutiques chez l'enfant, l'adolescent et l'adulte

\section{6-8 juillet 2005}

Faculté de Médecine Xavier Bichat, 16, rue Henri Huchard, 75018 Paris, France

\author{
Organisé par l'Association pour le Développement de la Recherche \\ sur l'Attachement (ADRA)
}

Organisation scientifique

Professeur Antoine Guedeney, Professeur Abraham Sagi-Schwartz, Docteur Nicole Guedeney

\section{Orateurs}

Karl Heinz Brisch (Allemagne), John Byng-Hall (Royaume-Uni), Jude Cassidy (USA), Mary Dozier (USA), Elisabeth Fivaz Depursinge (Suisse), Yvon Gauthier (Canada),

Nicole Guedeney (France), Erik Hesse (USA), Jeremy Holmes (Royaume-Uni), Kai von Klitzling (Suisse), Giovanni Liotti (Italie), Mary Main (USA), Robert Marvin (USA), David Oppenheim (Israël), Abraham Sagi-Schwartz (Israël), Daniel Stern (USA), Daniel Widlöcher (France), Charles H. Zeanah (USA)

\section{Renseignements}

BCA, 6, boulevard du Général Leclerc, 92115 Clichy Cedex, France

Tél. : 0141066770 - Fax : 0141066779

Email : contact@b-c-a.fr

Site : www.b-c-a.fr 